\title{
The AXS battery and "neurological fingerprints": Meeting the challenge of individual differences in human brain/behavior relations
}

\author{
J. L. LAUTER \\ University of Oklahoma Health Sciences Center, Oklahoma City, Oklahoma
}

\begin{abstract}
A new test battery, the Auditory Cross-Section (AXS) Battery, offers a relatively inexpensive, noninvasive means of describing a "neurological fingerprint" for each individual. The battery's "access to axes" combines physiological and behavioral measures so that a large set of dependent variables can be used to profile an individual and can serve as a context for additional anatomical, behavioral, and physiological data for the same subject. Physiological tests included in the battery described here include (1) otoacoustic emissions (OAEs); (2) the repeated evoked potentials version of the auditory brainstem response (REPs/ABR); and (3) quantitative electroencephalography (qEEG). Complementary behavioral tests were chosen to assess capabilities related to functional asymmetries, such as phonemic awareness and fine motor control, and/or to demonstrate temporal correlations that link behavioral and neural function. Applications of the AXS battery include (1) documentation of individual differences and similarities in neural organization that are related to specific behaviors, such as learning styles or clinical symptoms; (2) provision of contextual data for neuroimaging studies that aid in interpreting individually specific patterns of activation; and (3) development of a neurotypology of human brain/behavior relations, linking characteristics of neuroanatomy and neurophysiology with features of behavior, and general body health.
\end{abstract}

One of the greatest challenges in the study of neurological correlates of behavior in humans is the interpretation of the dramatic degree of difference across individuals. Such differences are found with virtually all sophisticated measures of human behavior, from behavioral assessments of psychophysical skills to noninvasive imaging of neuroanatomical and neurophysiological correlates of behavior. Yet the rich diversity of detail is often either ignored or is considered to be experimental noise and is, therefore, actively suppressed through the use of statistical methods that emphasize group rather than individual results.

Over the past two decades, research done in our Coordinated Noninvasive Studies Project has involved the exploration of the utility of a variety of noninvasive methods for studying individualized features of human brain/ behavior relations. The methods employed or considered have included: psychophysically controlled behavioral tasks such as dichotic listening (e.g., Lauter, 1982, 1983, 1984), evoked potentials (e.g., Lauter \& Karzon, 1990; Lauter \& Loomis, 1986; Lauter, Lord-Maes, \& Baldwin, 1999; Lauter \& Oyler, 1992), magnetoencephalography,

This paper is based on a presentation to the Measuring Behavior ' 98 Conference, August 1998, Groningen, The Netherlands. The AXS Battery represents an approach and recommended set of procedures. The commercially available hardware and software described here were developed by others, and we have received no financial consideration from the vendors to mention their specific products in this report. Address correspondence to J. L. Lauter, Center for Communication Neuroscience, University of Oklahoma Health Sciences Center, Oklahoma City, OK 73190 (e-mail: judith-lauter@ouhsc.edu). positron emission tomography (e.g., Lauter, Herscovitch, Formby, \& Raichle, 1985; Lauter, Herscovitch, \& Raichle, 1988), single-photon-emission computed tomography, functional and anatomical magnetic resonance imaging (e.g., Lauter \& Plante, 1992), event-related optical signals (EROS), and quantitative EEG (e.g., Lauter, 1992). The use of these techniques, from the simplest evoked potentials to the most expensive blood flow imaging, reveals a dramatic degree of individual differences in the details of brain structure and function. Thus we have concluded that accounting for the "repertoire" of individual patterns is a prerequisite for understanding the variety of ways in which human brain organization underlies behavior.

For financial reasons, the availability of most brain imaging technologies is highly restricted (Lauter, 1995). The majority of these technologies involve equipment that is expensive and requires special testing facilities. Their use is extremely personnel intensive and requires teams of individuals to assist with daily maintenance, data collection, and data analysis. As a result, many such methods, at least for the indefinite future, can be expected to remain beyond the means of the majority of researchers. Thus we have been motivated to develop a test battery that can provide a way of deriving individually specific neural profiles in a form that should be within the means of many more laboratories than can afford the equipment, facilities, and personnel costs associated with an MRI or a PET system.

In this article, we describe the design and use of a test battery that has emerged from this research, termed the Auditory Cross-Section (AXS) Battery. The AXS Battery 
provides a relatively inexpensive, noninvasive means of describing a representative "neurological fingerprint" for each individual, based on use of the auditory system as a microcosm of functional neural organization. The battery uses equipment that is commonly found in many types of laboratories and audiology clinics. The procedures for using the equipment are easily learned and include a set of protocols that can be conducted by one person. Just as with forensic uses of real fingerprints, the dependent variables yielded by the battery offer not only a means of uniquely describing each individual, but also an objective basis for classifying individuals into groups, providing support for formulations of universal principles of brain/ behavior relations. Such classification may be not only important in and of itself, but also crucial in providing the background for understanding individual differences that are observed in behavioral assessments and/or in experiments in which more expensive types of neuroimaging are used.

\section{PRINCIPLES BEHIND THE AXS BATTERY}

\section{Study Individuals, Not Groups}

In order to understand brain/behavior relations in humans, one needs to know more about the range and the nature of individual variation. Knowing individual details is an absolute prerequisite for classification and typology, whether the goal is to describe features of "normal" individuals, or to define diagnostic hallmarks of clinical categories. Population statistics are meaningful only after groups have been formed on the basis of multifaceted characterizations of individuals. Although experiments may be carried out according to a priori hypotheses as to grouping, procedures should be designed to generate data that can serve as the basis for new approaches to classification and the data generated must also allow grouping of subjects on an a posteriori basis. In this way, every experiment can provide a concrete stepping stone to the next new idea, rather than simply close the door on an old one.

\section{Many Angles of View \\ Should Be Used, Not Peepholes}

In order to characterize the neural correlates of behavior for each individual, it is necessary to create a unique profile, or "neurological fingerprint" for each individual tested. In order to create such a profile, features from three relevant categories must be combined. The categories are behavior (including clinical symptoms), anatomy and/or physiology at the body periphery, and anatomy and/ or physiology of the central nervous system (CNS). The features must be combined in a way that allows them to be related in a dynamic and interactive way. Whether in studies of normal function or dysfunction, it is predictable that when descriptions are limited to one system at a time, or one level at a time, or one local region at a time, it is impossible to fairly represent the whole organism or the bi- ological nature of behavior. It is crucial to understand that observable behaviors or symptoms are expressions of the biological fact that ultimately "everything is connected to everything else."

\section{Describe Neural Function in \\ Terms of Dynamic Axes, Not Static Sites}

A neurobehavioral profile for an individual must represent or provide the basis for representing dynamic "handshaking" relations along all three body/brain axes (right-left, rostro-caudal, and dorso-ventral; Lauter, 1999b). There are two basic assumptions about the nature of these relations. First, no axis involves "one-way" function only-rather, each is characterized by intrinsic "back-and-forth" interactions among its own components. Second, no axis functions in isolation from the othersextrinsic relations linking all three axes may mean that it is impossible to understand the organization of one without studying at least some aspects of the others.

\section{Build the Big Picture Via "Connecting the Dots," Not "Beads On a String"}

Data relating to the classes of features described above must be collected and analyzed in a way that allows results to be related to each other and be interpreted in the context of the whole data set. Therefore, test batteries must be designed in order to generate results that can be triangulated with each other, so that the findings from each component test can contribute to the gestalt of results from all the tests. This type of "coordinated" test battery, which is very different from a "beads on a string" approach, makes it potentially possible to draw sophisticated and wide ranging conclusions from relatively small amounts of data. This is an important feature, given the practical constraints discussed below.

\section{In the Real World, There Is Not Enough Time}

The first practical consideration addressed by the design of the AXS Battery is time. Although it would be ideal to fulfill the recommendations listed above by studying everything about each individual, this is obviously not possible, and even characterizations that fall well short of "everything" may be very difficult to achieve. The strategy represented by the AXS Battery is to select a single functional system to represent body/brain organization in the whole individual. The functional system we chose is the auditory system, which was selected for two reasons. First, the auditory system offers a microcosm of neural organization, which extends from the body periphery to the cortex, making it readily accessible at every level to quantitative study via relatively inexpensive methods. Second, the auditory system is directly related to many issues related to human behavior, such as speech perception and production, environmental stimulus processing, language acquisition and cognitive development, prenatal brain growth, response to brain injury, and psychiatric conditions. 


\section{In the Real World, There Is Not Enough Money}

The second practical consideration addressed by the AXS Battery is the cost of research. Although it would be ideal to study brain/behavior relations in each individual with every available noninvasive tool for behavioral, physical, anatomical, and physiological assessment, the economics of research make this impossible. The costs associated with infrastructure, purchase, maintenance, and personnel support of many noninvasive methods are prohibitive for the vast majority of researchers interested in the human brain. Therefore, the AXS Battery requires a selected set of test instruments that can be purchased, maintained, and used within a modest budget, and can still satisfy the criteria for the research methodology described above.

As utilized in our laboratory, conducting the AXS Battery begins with a basic set of behavioral measures and proceeds to a series of physiological assessments, which are designed to represent a "cross-section" of processing in the auditory system, from periphery to cortex. The minimal set of behavioral tests includes a detailed family and personal medical history; audiometric assessment of outer, middle, and inner ear function, including tests of puretone and speech perception; and a series of measures that relate to functional asymmetries, such as phonemic awareness, fine motor control, and geometric figure reproduction. Additional behavioral tests may be conducted in order to document within-subject changes over time, such as occur before and after behavioral or pharmaceutical challenges, or clinical treatments.

The physiological tests of the AXS Battery include the following: otoacoustic emissions (OAEs), the repeated evoked potentials version of the auditory brainstem response (REPs/ABR), and quantitative electroencephalography ( $\mathrm{qEEG}$ ), which is conducted under resting conditions. Otoacoustic emissions are air-propagated sound waves that can be recorded by a microphone placed in the outer ear canal (see Probst, Lonsbury-Martin, \& Martin, 1991). They are considered to be the result of movements of outer hair cells that are located within the cochlea; the majority of innervation from the hair cells is efferent rather than afferent. Thus OAEs provide a means of examining a type of peripheral sensory function that is not only specific with regard to side (right vs. left), but may also reflect top-down influence of the CNS on the auditory periphery, a possibility supported by preliminary findings using the AXS Battery (see Lauter, 1998b; Lauter, Lynch, Wood, \& Schoeffler, 1999).

The ABR represents evoked electrical activity that is recorded via scalp electrodes and is generated by portions of the auditory system that extend from the auditory nerve (cranial VIII) to the midbrain (see Hall, 1992). The REPs protocol for ABRs developed in our laboratories is based on minor modifications of standard ABR testing techniques and is designed to support repeatability analysis of waveform parameters such as latency and ampli- tude. The protocol provides information on responses during right-ear, left-ear, and binaural stimulation conditions. To date, the findings obtained using the AXS Battery support the conclusion that waveform repeatability can serve as a basis for evaluating efferent as well as afferent interactions within a sensory pathway (Lauter, Brand, Messina, \& Perkins, 1998; Lauter, Lynch, et al., 1999; Lauter, Richey, Gilmore, \& Lynch, 1998).

In order to represent cortical activity, the AXS Battery collects "running EEG" data under resting conditions. This is done in order to estimate the degree of underlying anatomical, hemispheric asymmetry in comparing left- and right-side auditory cortex (see Plante, Swisher, Vance, \& Rapcsak, 1991), a feature which has been linked in previous observations (see Galaburda, Rosen, \& Sherman, 1990; Plante et al., 1991) to a number of features, such as handedness and language abilities. Previously, we have shown that in neurologically normal subjects, resting qEEG asymmetry can predict the anatomical asymmetry (measured with MRI) and that a mismatch between anatomy and resting qEEG activity may be diagnostic (Lauter, Richey, et al., 1998).

The qEEG provides information about local EEG power (via spectral analysis) and about the nature of interaction (measured as "coherence") between the two sides (see Zappulla, 1991). The results can be interpreted not only in terms of the characterization of activity at the level of the cortex, but also the ways in which this relates to the findings for the auditory brainstem and the ear measured in the REPs/ABR and OAE subtests described above. Although the AXS Battery currently does not make use of other EEG-based measures, such as cortical evoked potentials (EPs), the dramatic individual differences commonly observed in EPs at this level might usefully be interpreted against the background of the basic measures provided by the battery (see Lauter \& Karzon, 1990).

Data from the AXS Battery can also serve as a useful context for other information on individual subjects, such as additional behavioral measures; MRI documentation of features such as the anatomical integrity of cerebral structures and volume asymmetries of specific brain regions such as perisylvian cortex; and data derived from physiological imaging techniques such as SPECT, PET, fMRI, and EROS.

The "bottom-up" approach in the design of this battery is derived from the conviction that the focus on the range of individual expressions may lead to principles of brain/behavior classification that could be derived in no other way. One example is our trimodal model of brain organization (Lauter, 1998a, 1999b, in press), which posits the existence of a trimodally distributed continuum of individual differences that are based on hormonal exposure during prenatal development, with behavioral expression being based on individual differences in the salience of right- and left-hemisphere functional asymmetry capabilities. The AXS Battery offers a practical 
means for the collection of individually specific types of data that are required for developing and testing the neurotypology of human brain/behavior relations.

\section{FACILITIES AND EQUIPMENT REQUIRED FOR THE AXS BATTERY}

In this section, we describe both the optimal and minimal requirements for conducting tests with the AXS Battery.

\section{Facilities}

The best facility for conducting the AXS Battery and the accompanying audiometric evaluations would provide four sound-treated, two-room test suites, with at least two of the test rooms electrically shielded. This configuration is currently in use at the Center for Communication Neuroscience (CCNS). One of the sound-treated suites is used for audiometric testing, the two electrically shielded rooms are dedicated to electrophysiological testing (one for EPs, one for qEEG), and the fourth suite is utilized for the testing of OAEs. However, it is possible to collect reliable AXS data in a single, non-sound-treated room, as long as the room has properly grounded outlets, can be kept very quiet, and has minimal electrical interference.

\section{Equipment}

The AXS Battery requires computer-based capabilities for collecting and analyzing the following tests: (1) OAEs--at least one type of evoked emission is needed (see below); (2) auditory evoked potentials (aEPs) -at least 2-channel capability for collecting the family of auditory EPs from brainstem to cortex (note that no "oddball paradigms" are required); and (3) qEEG - at least 4channel recording capability, with software routines for spectral and coherence analysis. Research is currently underway in the CCNS laboratories in order to determine whether a system capable of conducting cortical EPs can be used to provide information similar to that derived from qEEG, such as the direction and magnitude of physiological asymmetry comparing right- and left-side auditory cortex. However, until that research is complete, 4channel qEEG remains a crucial part of the AXS Battery.

The optimal configuration assigns the three AXS tests to three separate computer systems, located in three independent test suites, as described above. In our CCNS laboratories we employ an Otodynamics ILO-92 system for collecting OAEs, a Biologic Traveller for EPs, and a Neuroscan 32-channel qEEG system for recording and analyzing qEEG data. However, it is possible to have all three of these capabilities on a single computer system, if the proper computer and add-on components are selected. For example, all current $q E E G$ vendors offer a variety of EP selections on the same system, and, although no $\mathrm{qEEG}$ package currently includes an OAE option, add- on hardware and software may be purchased in order to add OAE capability to an existing qEEG or EP system.

For the most complete audiometric analysis, a professional quality audiometer is needed that has capabilities for pure-tone and speech testing, as well as an impedance bridge for physiological evaluation of middle-ear function. More minimally, a portable audiometer can provide threshold values for a sampling of pure-tone frequencies, which is necessary for interpreting results on the AXS Battery.

\section{DATA COLLECTION PROCEDURES}

In this section, we briefly describe a typical session in which an individual subject is tested with the AXS Battery as it is implemented in the CCNS laboratories.

\section{Consent Form and Audiometry}

At the beginning of the test session or beforehand, a consent form is presented and signed, a medical history is taken, and an audiometric evaluation is performed; this includes tympanometry, detection thresholds in each ear for pulsed pure tones from 250 to $1200 \mathrm{~Hz}$ and speech discrimination thresholds for spondee words. If time permits, one or more central auditory tests may also be given (such as the SCAN [Keith, 1986], the Staggered Spondaic Word [SSW] test [Katz, 1986], or the speech/nonspeech components of MacCAD [Lauter, Solomon, \& Lord-Maes, 1991]), to provide additional information on the auditory perception of each subject.

\section{Electrode Placement}

For a minimal battery run that includes REPS/ABR and 4-channel qEEG, seven 9-mm disk electrodes are placed on the head of the subject at the following sites: forehead (ground), just over the left eyebrow (eye movement monitor for $\mathrm{qEEG}$ recording), right and left earlobes (references), 1 in. above the top of each pinna (standard sites $\mathrm{T} 3$ and $\mathrm{T} 4$, which serve as active electrodes that represent the left and right auditory cortex, respectively), and the top of the head (vertex - active electrode for both ABRs and qEEG). Impedances are checked periodically during the test session and are maintained below $5 \mathrm{k} \Omega$ at all times.

\section{Otoacoustic Emissions}

In the CCNS laboratories, OAE testing is done with an ILO-92 system that is run on a PC. The computer is located in the outer control room of the two-room sound-treated test suite, with the interface box set on a table next to the subject in an inner test room. The subject is seated in a comfortable reclining chair. A soft foam tip is prepared for the earprobe, which is placed in the outer ear canal (the right ear is tested first), where the fit should be snug but comfortable. The lead to the probe is adjusted so that it rests on the back of the reclining chair and not on the sub- 
ject's shoulder, so that movements of clothing against the probe lead, such as may occur during quiet breathing, do not introduce perceptible noise into the probe.

Each subject is informed about the details of OAE testing in order to ensure full cooperation during the procedure. The subject is instructed to sit with eyes closed and to remain still and quiet throughout the test. The subject is told that movements, including swallowing, are acceptable during any silent time, but that when sounds can be heard in the probe, movements should be held to a minimum, because the tiny microphone in the earprobe is very sensitive to the noise that such movements create inside the ear canal.

Spontaneous OAEs are collected first, followed by the transient-evoked "growth" routine, in which clicks are presented at four different levels around a baseline of $75 \mathrm{~dB}$ SPL. Then a repeated measures series of distortionproduct OAEs are collected. Our CCNS system uses a macro for DPOAEs which presents pairs of primaries at $70 \mathrm{~dB}$ SPL, with a 1.2 frequency ratio, in 26. steps ranging from $F 1=708 \mathrm{~Hz}$ to $F 1=6165 \mathrm{~Hz}$. Each pass through the series is saved as a separate file in order to support repeatability analysis (see the Data Analysis section below). The complete set of SOAE, TEOAE, and DPOAE tests for the right ear requires about $10 \mathrm{~min}$, and it is then repeated in the left ear.

\section{qEEG Testing}

In the CCNS laboratories, qEEG testing is done using a Neuroscan 32-channel desktop system with Neuroscan's SYNAMPs, running on a pair of PCs. The computers and amplifiers are located in the outer control room of a two-room sound-treated test suite, with the interface box set on a table next to the subject in an electrically shielded inner test room. The subject is seated in a comfortable reclining chair, and the electrodes are checked for impedances.

Once impedances are found to be satisfactory, the subject is informed about the details of qEEG testing, to ensure full cooperation during the procedure. The subject is told that the resting condition will take only about $5.5 \mathrm{~min}$, during which time it is necessary to remain still and quiet with eyes closed throughout the test. The subject is reminded that movements, including swallowing, should be held to a minimum, because the electrodes are sensitive to any type of muscle activity around the head and neck. The subject is encouraged to relax the jaw and shoulder muscles and is told to remain awake and alert with eyes closed.

The basic AXS qEEG recording is designed to represent the pattern of resting activity at the vertex and over each side of auditory cortex; therefore, no type of stimulation is given during the $5.5-\mathrm{min}$ data collection. Room lights in the test room are darkened, the test room door is closed, and qEEG is recorded for approximately $5.5 \mathrm{~min}$. The subject is then given the chance to take a break, since the final physiological component of the test session (REPs/ABR) requires $45-50 \mathrm{~min}$ to complete.

\section{REPs/ABR}

In the CCNS laboratories, REPs/ABR testing is done with a Biologic system running on a PC. The computer is located in the electrically shielded inner test room of a two-room sound-treated suite, so that the experimenter's back is to the subject, who is seated in a comfortable reclining chair. Electrodes are connected to support simultaneous 2-channel recording (vertex to each earlobe), and impedances are maintained below $5 \mathrm{k} \Omega$ at all times.

The subject is informed about the details of REPs/ABR testing, to ensure full cooperation during the procedure. The subject is asked to sit with eyes closed and to remain still and quiet throughout the test. Ideally, the subject would go to sleep during this test, because, as with qEEG, movements about the head and neck need to be held to a minimum. Relaxing the jaw and shoulder muscles is the best way for the subject to assist in the testing. Five waveforms are collected with clicks in the left ear (the first is usually omitted from analysis), followed by four waveforms with clicks in the right ear, then eight waveforms with binaural clicks. Prior to binaural stimulation, the earlobe electrodes are linked at the transducer box.

Note that the REPs/ABR testing must be scheduled as the last physiological test in the AXS session, because of the level of stimulation associated with this test. Informal observations have indicated that both OAEs and qEEG test results are different when these tests are done after, rather than before, REPs/ABR testing.

\section{Functional Asymmetry Behavioral Battery}

A variety of tests could be used for this part of the battery. Currently, in our CCNS laboratories, we administer a "sidedness" questionnaire, a test (e.g., a pegboard test) of fine motor control, and assess phonemic awareness via the Lindamood Auditory Conceptualization (LAC) Test (C. Lindamood \& P. Lindamood, 1979; P. Lindamood, Bell, \& P. Lindamood, 1997). We have found that phonemic awareness may be related to cortical asymmetries (see Lauter, 1998a). Behavioral tests that are used to assess changes in performance over time include such things as the recording of speech productions and computer-based assessments of eye-hand coordination and eye movements.

\section{DATA ANALYSIS GUIDELINES}

The time that it takes to retrieve the raw data for the battery is approximately the same as the time that it takes to administer it. Additional analysis, graphing of results, and preparation of a narrative report for each subject can take several hours.

\section{OAEs}

For each subject, data tables are made, which show a variety of values for each ear, such as (1) frequency and amplitude of each SOAE identified, (2) amplitudes of TEOAE components, and (3) amplitude and amplitude standard deviation of DPOAEs. 


\section{REPs/ABRs}

For each subject, data tables are made, which show values for latency and amplitude of the first six vertex positive peaks in the ABR waveform and also show calculations of the mean and standard deviations of these values over a series of four waveforms.

For analysis, the five left-ear waveforms are displayed and a decision is made as to which four will be used for analysis (usually the first one is discarded, allowing the subject's system a chance to "settle in" to the procedure; if another waveform has much higher artifacts, or an unusual morphology as compared with the others, it can be discarded instead). The four left-ear waveforms to be analyzed are then redisplayed and the screen cursor is utilized to identify peaks and to retrieve latency and amplitude values for each, followed by peak identification for right-ear and binaural waveforms.

Peak identification procedures that are used for the AXS Battery are designed in order to avoid the subjectivity that is implicit in many EP measurement methods. This approach utilizes the highest amplitude point in each peak to represent that peak. The set of rules employed also addresses instances in which several points have the same amplitude, or in which there is no valley separating peaks. This makes it possible to achieve near-perfect interjudge and intrajudge repeatability, even for inexperienced observers.

Once all latency and amplitude values for all six peaks tested under all three ear conditions have been entered, calculations for each set of four repetitions provide the mean, the standard deviation, and the coefficient of stability $\left(C_{\mathrm{s}}=X / S D\right)$. In later stages of analysis of REPs/ABR results, the mean and $C_{\mathrm{s}}$ values prove to be most useful. These values can be employed in a variety of ways - for example, to graph profiles that compare the three ear conditions in terms of absolute latency, absolute amplitude, latency stability, and amplitude stability and to compare the first four binaural waveforms with the second four binaural waveforms. Examples of findings that have resulted from this approach have been reported in a series of presentations and publications that date from the early 1980s (e.g., Lauter \& Karzon, 1990; Lauter \& Loomis, 1986; Lauter, Lynch, et al., 1999; Lauter \& Oyler, 1992).

Such comparisons not only provide a quantitative means of displaying the "fingerprint" features of each subject, but often are very helpful in characterizing an individual system for comparisons with the other tests in the battery, and/or for help in clinical interpretation. For example, individuals who have more activity in one side of the auditory cortex often show higher amplitude for the contralateral-ear response at ABR peak III. Or, a subject with late-stage multiple sclerosis may show a sharp increase in the latency stability of one or another ABR peak if one compares the second four with the first four binaural runs, which suggests a failure in top-down modulation in response to the "stress" of continued testing under the same condition (Lauter, Lord-Maes, \& Baldwin, 1999).

There are additional stages of analysis that can be done with these data; however, space does not permit detailing those here (examples have been presented for a number of applications-for additional discussion, see Lauter, Richey, et al., 1998). One example, our "REPs score" procedure, is briefly described here. For each laboratory where such testing is done, one can prepare a comparison database that represents "normative" data for latency and amplitude mean and $C_{\mathrm{s}}$ values, and then score the values for each new subject against this database. The resulting comparisons can be interpreted in terms of cases in which the new data fall within a range (e.g., 1-3 SDs) of the database compared with those in which new data fall outside such a range, either below or above the normative value.

In our labs, we have developed a Macintosh-based scoring program in order to make such comparisons automatically. Means and $C_{\mathrm{s}}$ values are entered, and the program scores the instances in which individual values fall outside the designated range as "REP scores," which represent deviations from the normal database. REP scores in which latency is later than normal, or amplitude/latency stability/amplitude stability are lower than normal, are called "negative REP scores" and are interpreted as "Jacksonian loss signs," which are analogous to flaccid paralysis in the motor system and reflect problems at or below the peak where they are expressed. Thus damage to the cochlea or eighth nerve might result in diminished amplitude and/or latency stability of ABR peak I.

REP scores with values "better" than normal (i.e., latency is earlier, or one of the other three measures is larger than the comparison values) are called "positive REP scores" and are interpreted as "Jacksonian release signs," which are analogous to spastic paralysis and indicate a failure of top-down modulation by centers above the peak where they are expressed. Thus, extremely high latency stability of peak III during right-ear testing might suggest a failure in top-down modulation by either the left-side midbrain or the left-side auditory cortex. (For further discussion, and other clinical examples that cite REP score values and interpretations, see Lauter, LordMaes, \& Baldwin, 1999; Lauter, Richey, et al., 1998).

The findings reported to date suggest that conducting ABR testing under the REPs protocol, may make it possible to assess right-left and rostro-caudal relations, not only within the brainstem itself (e.g., modulation by peak-V generators on peak III), but also extending below and above the brainstem (e.g., sensorineural hearing loss degrading ABR values, or a failure in cortical downmodulation on ABR peaks). For example, REPs/ABR can be used to document two-stage effects of an antivertigo antihistamine, such as meclizine, which directly affect the cortex and some portions of the brainstem, and is accompanied by delayed "waterfall" effects on more caudal re- 
gions, documented by changes in stability from level to level of the response (Lauter, Lynch, et al., 1999).

\section{qEEG}

The "quantitative" part of qEEG is the key to analysis of EEG data. Data analysis packages, which should be available on the recording system, are used to generate a table for each subject. The table shows power (in microvolts squared) in each of four standard qEEG bands at each active electrode site (typical bands are $1-3.9 \mathrm{~Hz}=$ delta, $4.0-7.9 \mathrm{~Hz}=$ theta, $8.0-11.9 \mathrm{~Hz}=$ alpha, $12.0-15.9$ or $19.9 \mathrm{~Hz}=$ beta), coherence (expressed as percent) for each of the qEEG bands, comparing pairs of active sites (e.g., vertex vs. T3, vertex vs. T4, and T3 vs. T4).

Interpretation of these values can be based on the cortical findings alone. For example, in previous research, we have found that the power asymmetry between leftside and right-side auditory cortex (calculated as the difference divided by the sum, multiplied by 100 ) provides a good estimate for many subjects, of the direction and magnitude of the underlying anatomical perisylvian asymmetry, as could be measured by MRI (Lauter \& Plante, 1992; Lauter, Richey, et al., 1998). In addition, comparisons can be made with other findings in the AXS Battery. These include comparisons between cortical power asymmetry and the stability of DPOAEs in the right and the left ear (Lauter, 1998b), or cortical coherence and the stability of ABR peak V, and so forth.

The power asymmetry in the auditory cortex can also be used to illuminate the physiological basis of individual differences in assessed behaviors, such as performance on the functional asymmetry battery described above. For instance, individuals for whom there is a qEEG power asymmetry that favors either side of the auditory cortex (either a left-hemisphere advantage [LHA] or a righthemisphere advantage [RHA]) perform well on the LAC test of phonemic awareness, whereas those who exhibit a condition of power symmetry in the auditory cortex often do not do as well on the LAC test (Lauter, 1998a). This finding is related to findings of other authors (for reviews, see Galaburda et al., 1990; Plante et al., 1991), who have concluded that a condition of anatomical symmetry in perisylvian regions-including areas overlain by $\mathrm{T} 3 / 4$ scalp electrodes - is often associated with reading problems, which are potentially based on fundamental difficulties with aspects of auditory processing, such as phonemic awareness.

It is also possible to use the qEEG asymmetry value as an estimate of a "resting physiological bias" in order to aid in the interpretation of individual differences in "ear advantage" scores, such as those generated by dichotic identification of speech and nonspeech sounds. For example, dramatic individually specific distinctions in patterns of "absolute ear advantages" and "relative ear advantages" (Lauter, 1982, 1983, 1984) may be in part determined by the underlying right-left bias of individ- ual systems which can be expressed in both anatomical (Lauter \& Plante, 1992) and physiological (e.g., qEEGsee Lauter, 1992) terms.

\section{IMPLICATIONS FOR UNDERSTANDING HUMAN BRAIN/ BEHAVIOR RELATIONS}

We believe that the AXS Battery has the potential to serve as an important tool in studies of human brain/ behavior relations for the following reasons: (1) The battery generates data that can be used to create a "fingerprint" - that is, a specific neural profile for each individual; (2) the resulting profile represents a systems-level characterization that provides details related to each single level and side of the system within a larger context of information that is useful for comprehending how the components of the system are related in a dynamic, "handshaking," way; (3) these systems-level neural profiles can be readily compared with individual ratings on any type of behavioral scale or measure; (4) the individual profiles are sufficiently detailed and complete to offer potentially valuable complements to results that have been collected for the same subjects using other noninvasive methods, such as anatomical or blood-flow images; and (5) the infrastructure and training that are required for implementing the battery are minimal, particularly compared with many other types of neuroimaging methods, and thus may render this approach to neural testing accessible to a larger number of researchers.

The coordinated nature of the battery, and the type of data collected, combined with the strategies for analysis procedures, make the battery feasible for studying virtually every aspect of brain/behavior relations in "normal" individuals, as well as in those with developmental and/or acquired disorders. For clinical populations especially, the dynamic, organizational picture of brain organization that the battery provides holds promise for replacing the static, phrenological, or "lesion-oriented," model of dysfunction, which has proven to be of limited utility for assessment of individuals presenting with diagnoses such as developmental disorders or psychiatric conditions, which may have even retarded our understanding of individual differences in response to acquired injury or to therapy.

In our CCNS laboratories, we have used the AXS Battery to examine neural correlates of a wide variety of individual differences in features such as performance on dichotic-listening tests (Lauter, 1992), susceptibility to chronic pain such as phantom limb and temporomandibular joint pain (Lauter, Brand, et al., 1998), eye-movement characteristics, handedness, stages of multiple sclerosis (Lauter, Lord-Maes, \& Baldwin, 1999), abilities in phonemic awareness (Lauter, 1998b), degree and nature of nicotine addiction (Lauter, Lynch, et al., 1999), response to oto- and neurotoxic chemicals, central auditory processing abilities (Lauter, Richey, et al., 1998), sensitivity to 

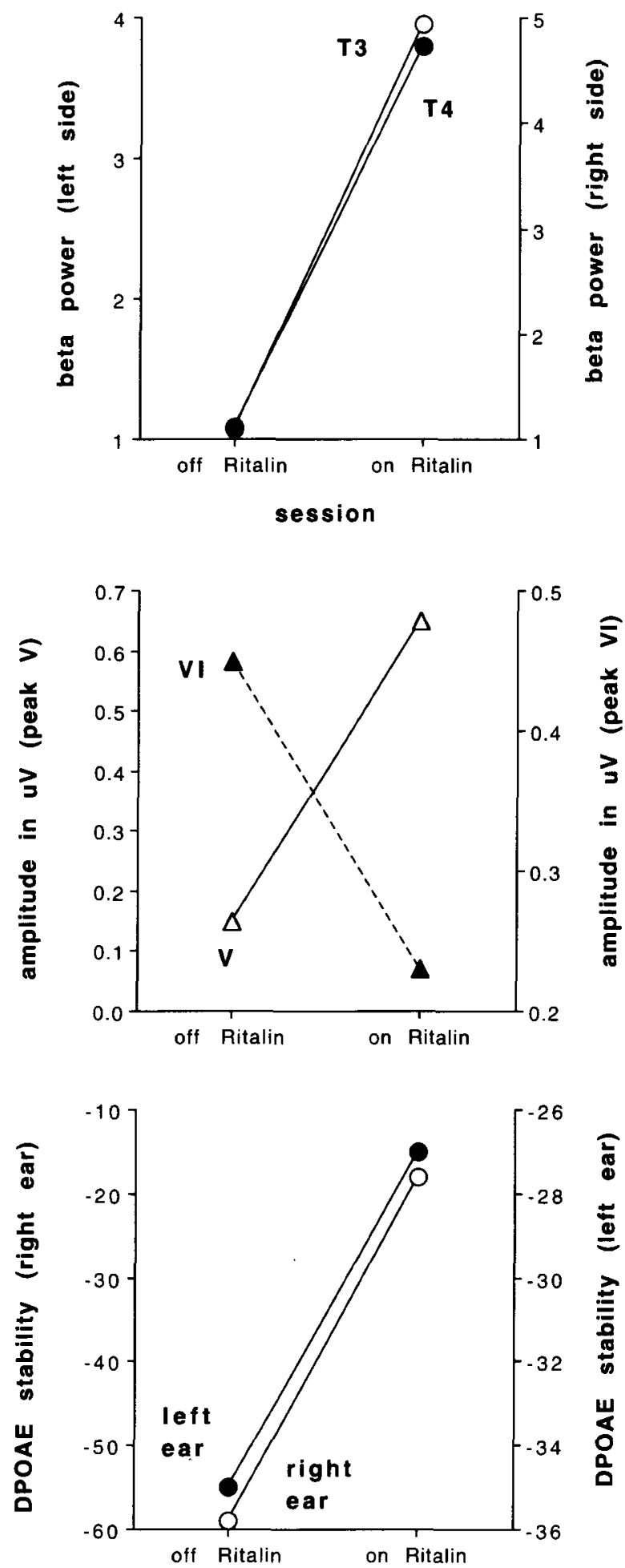

Figure 1. Sample results from a within-subject study of changes in response to Ritalin. Data displayed include qEEG measures of beta power over left- and right-side auditory cortex (top); amplitude of ABR peak VI compared with peak V (middle); and the amplitude stability of distortion-product otoacoustic emissions (DPOAEs) in right and left ears (bottom). Changes observed in brainstem and ear are interpreted as consistent with Ritalin's direct effect on the cortex, and, thus, as documenting a "see-saw" series of loss/release changes along the rostro-caudal neuraxis. antivertigo medications (Lauter, Lynch, et al., 1999), cardiac function (Lauter, 2000), vocal motor control (Lauter, 2000), autonomic response to stressors, fine motor control, and personality.

To illustrate the general applicability of the AXS Battery, three examples from our within-subject studies of individuals are described: An "off-on" series of two sessions that document the effects of Ritalin on a child at the end and at the beginning of his normal dosage cycle, an "off-on-off" series of three sessions for an individual who reported hypothermia and cardiac symptoms in response to the fumes of an industrial chemical, which were retained in a piece of clothing, and a seven-session series conducted over a total of 10 days, designed to study withinsubject changes from periphery to cortex, due to spontaneous fluctuations in the nervous system.

The results of the meaurements related to Ritalin are illustrated in Figure 1. As is shown in the top panel, this boy's usual $10-\mathrm{mg}$ dose of Ritalin increased beta power over the two sides of auditory cortex (locations T3 and T4). This increase in activity at the cortical level is interpreted as being consistent with the series of observed "see-saw" subcortical changes (middle panel), where the amplitude of ABR peak VI decreased following Ritalin administration, and the amplitude of (presumably more caudal) peak $V$ increased. This "see-saw" form of level-to-level changes resembles a pattern observed in an earlier study of the effects of an antivertigo antihistamine (Lauter, Lynch, et al., 1999), where peak-to-peak alternations of up regulation and down regulation from top to bottom of the brainstem were interpreted as representing successive "loss-release" interactions along the rostro-caudal neuraxis.

In the present case, the alternating effect culminates at the auditory periphery in increased stability of distortion product OAEs (bottom panel), which is interpreted as being a sign of release of outer hair cell responsivity from top-down efferent regulation.

Results for the study on chemical exposure are illustrated in Figure 2. The individual in the study came to our laboratory for assistance in documenting his reaction to a new chemical being used in the plant where he worked. He reported that he had experienced hypothermia (core temperature down to $94^{\circ} \mathrm{F}$ ) and cardiac symptoms (accelerated pulse) in the factory. He had also noticed mild forms of the same reaction when he had been exposed to a jacket that had been worn in the plant. $\mathrm{He}$ asked if we would be willing to conduct in-laboratory testing on him before, during, and after exposure to the jacket.

A series of three sessions on the same day was scheduled and he was examined with two components of the AXS Battery--REPs/ABRs and OAEs (qEEG was not available at that time). The first session was run in the morning, the second immediately afterward, while he held the jacket on his chest and inhaled its quite noticeable fumes, and the third session followed a 90-min break, during which he walked about outside on a cold winter afternoon. His temperature was monitored periodically throughout the session. Although his temperature did not fall below $98.6^{\circ} \mathrm{F}$ at any time, he noted feeling "chilly" 

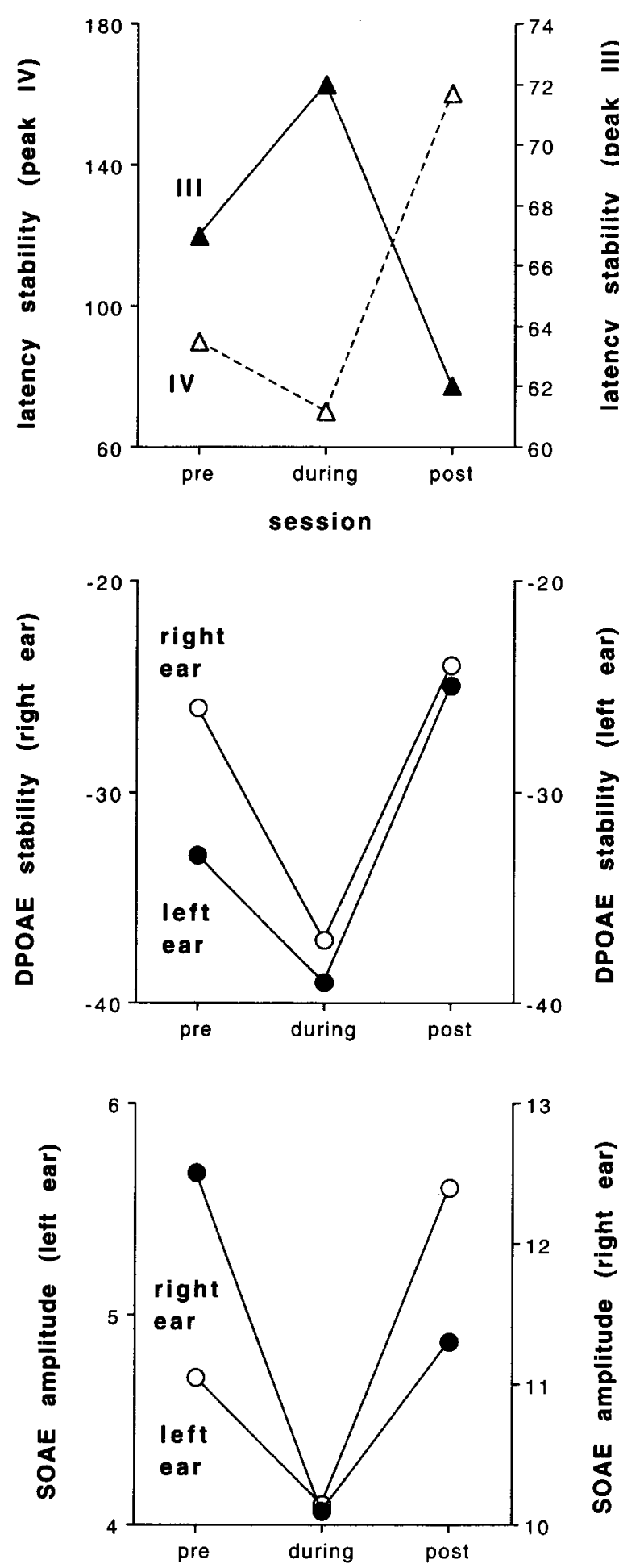

Figure 2. Sample results from a within-subject study of changes in response to challenge with an industrial chemical (see text for details). Data displayed include measures of ABR peak-latency stability for peaks III and IV (top); and assessments of changes in the ear, including the amplitude stability of DPOAEs (middle panel) and spontaneous emissions (bottom). Since qEEG was not available in this case, it is not known whether the changes in brainstem and ear were direct effects of the chemical, or were indirect, "downstream" physiological changes associated with a direct neurochemical action on the cortex. during exposure to the jacket and reported a concomitant tightness in the chest.

The top panel of Figure 2 shows a "see-saw" change over the course of the three sessions in response stability at ABR peak IV (destabilization occurs during exposure; this is followed by recovery with an "overshoot" value) and III (the opposite pattern). The two lower panels illustrate associated effects within the ear, documented via OAEs - changes in the stability of left- and right-ear DPOAEs (middle panel), and in the amplitude of spontaneous OAEs, one in each ear (bottom panel). Examination of the complete brainstem series in this individual suggests that effects of the chemical began at least at the level of the midbrain, since peak $\mathrm{V}$ decreased in stability during exposure and did not recover to baseline by the onset of the last session. Owing to the lack of qEEG in this case, it is not known whether the effects on peak $V$ were direct, or whether they were an indirect response to a more direct action on centers above the brainstem (i.e., thalamus or cortex). In any case, the changes from point to point along the brainstem were interpreted as being consistent with the hypothermia and cardiac symptoms that had been experienced during more intense exposure in the workplace.

Finally, Figure 3 illustrates findings for an individual who was tested in order to observe periphery-to-cortex patterns of activity in a series of Monday-WednesdayFriday sessions lasting approximately 10 days. In each session, the following measures were taken, in this order: (1) speech productions in order to represent laryngeal (sustained vowels) and upper-articulatory function (repeated stop-consonant syllables - ba-da-ga-pa-ta-ka) (assessed with a Kay CSL system that included electroglottography); (2) heart rate and diastolic/systolic pressure; (3) OAEs; (4) qEEG; and (5) REPs/ABR. The results shown in Figure 3 document changes over time at the level of cortex (top panel), contrasting cortex with upper brainstem (middle panel), and comparing two of the peripheral measures, voice pitch and DPOAE stability in the right ear (bottom panel).

The striking bilateral increase in cortical beta power that was observed on the first Friday (top panel) was reflected in a sharp downturn of stability at the level of ABR peak V (middle panel) and was apparently paralleled by increases in voice pitch and DPOAE stability (bottom panel). Although this study was designed to document dayto-day changes that were due to "spontaneous" neural fluctuations, questioning of the subject revealed that she had started taking antihistamines for allergies at the end of the first week (F1). The resulting sharp increase in cortical power seen on this day closely resembles the changes that had previously been documented in our meclizine study (Lauter, Lynch, et al., 1999); these changes were associated in that study with increased DPOAE stability, just as occurred here.

These three instances exemplify the broad-spectrum applicability of the AXS Battery and suggest its utility for many types of research. The most obvious application is the study of the neural correlates of individual differences in behavior, whether they involve normal or disordered 

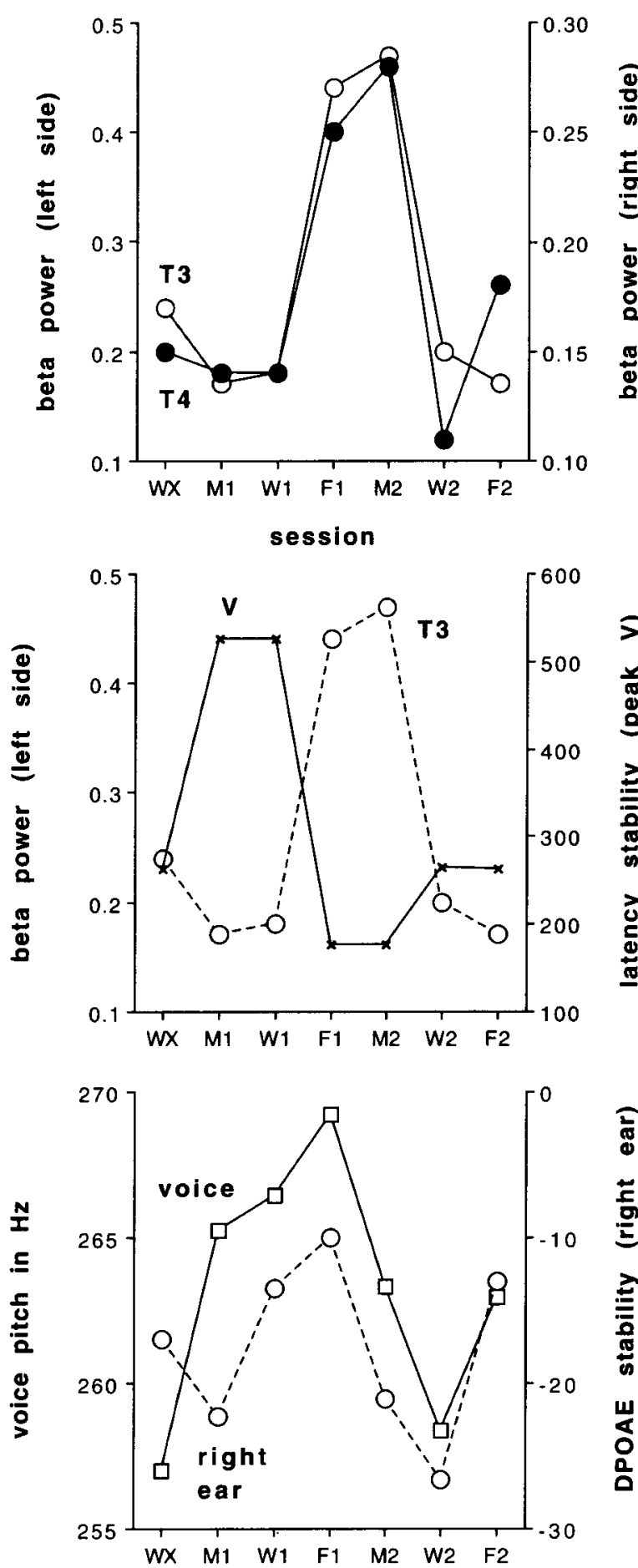

Figure 3. Sample results from a within-subject study assessing changes from periphery to cortex reflecting spontaneous day-today fluctuations. Data displayed include qEEG measures of beta power over left- and right-side auditory cortex (top); contrast of cortical data with latency stability of $A B R$ peak $V$ (middle panel); and comparison of changes in voice pitch and DPOAE amplitude stability (bottom panel). Patterns observed from level to level are interpreted as documenting dynamic relations that link cortex, brainstem, and the body periphery, and illustrate the utility of employing the auditory system as a microcosm to study central factors influencing nonauditory functions, such as aspects of motor control. behavioral features. Examples of characteristics that could be studied in this way include clusters of skills; the phenomenon of isolated skills; clinical signs such as the symptomatic behaviors of developmental conditions such as autism, hyperactivity, or dyslexia, or of psychiatric entities such as schizophrenia and depression; and changes in behavior associated with brain disorder or injury, such as perceptual dysfunction or aphasia.

For example, individual features could be defined via the AXS Battery soon after injury, and spaced retests could be used to follow the progress of spontaneous recovery, or to document response to therapy. In progressive disorders, the initial profile on the AXS Battery could be compared with later retests to document the extent and nature of change, and/or to compare vectors of disease progression across individuals. In all these instances, the AXS Battery has the potential to provide sufficient data on the neural characterization of each individual that can support correlations between behavior and aspects of neural organization that underly that behavior.

The whole-individual profiles provided by the AXS Battery could serve as a potentially valuable complement to other means of monitoring and imaging the nervous system, from MRI studies of brain anatomy to physiological studies of highly local patterns of activation measured with techniques such as blood-flow imaging (PET, SPECT, fMRI), or other means of monitoring brain activity (MEG and EROS). Often in such studies, the high resolution of the imaging technique reveals a striking degree of individually specific detail, which can be difficult to interpret in the absence of additional, related information regarding the same subjects. By pretesting each individual with the AXS Battery, imaging researchers might be able to generate a multidimensional context for their new data, and by this means find a way to exploit the individually specific resolution of their methods rather than have to degrade that resolution by averaging experimental results over individuals.

The more information we gather about the range of normal variation in human brain/behavior relations, the closer we may come to formulating a sophisticated neurotypology of human brain organization. Noninvasive methods have provided new information regarding aspects of individual anatomy, physiology, and even chemistry (Nyborg, 1994a, 1994b), which can be used to characterize both the somatic and the autonomic nervous systems (see Porges, 1995; Wittling, 1995). When these are combined with detailed behavioral observations of the same subjects, it may be possible to describe variants of human brain/behavior patterns, which could explain everyday observations of individual differences, gender differences, and distinctions regarding the propensity for certain skill profiles and/or susceptibility to certain disorders.

One example of such a formulation is the trimodal model of brain organization (Lauter, 1998a, 1999b, in press), which posits a continuum of individual differences expressed in terms of the relative salience of access to right- and left-hemisphere specializations and is shaped by prenatal exposure to gonadal hormones. The model predicts that this continuum is trimodally distributed in 
the greater human population and has implications for a wide range of covarying features that link brain anatomy, physiology, behavior, and general body health. All predictions of this model are eminently testable by means of methods like the AXS Battery and the collection of epidemiological data.

Certainly, for any of these applications, use of the AXS Battery to characterize individuals should be complemented whenever possible by results from other types of neural and behavioral testing, such as using MRI to identify anatomical features related to hormone-sensitive aspects of prenatal development as well as other means of physiological imaging, such as blood-flow imaging, in order to explore further the physiological patterns revealed by the AXS results. Extending the context of the battery in this way should not only reveal additional details about the nature of the topics under study, but should also help us understand more about the quality of information provided by the AXS Battery tests and protocols themselves. Further development of the battery will, it is hoped, extend its utility even further as an inexpensive and effective tool for human neuroscience.

\section{REFERENCES}

Galaburda, A. M., Rosen, G. D., \& Sherman, G. F. (1990). Individual variability in cortical organization and its relationship to brain laterality and implications of function. Neuropsychologia, 28, 529-546.

HALL, J. W., III (1992). Handbook of auditory evoked responses. Boston: Allyn \& Bacon.

KATZ, J. (1986). The SSW test manual (3rd ed.). Vancouver, WA: Precision Acoustics.

KEITH, R. (1986). SCAN: A screening test for auditory processing disorders. San Diego: Psychology Corporation.

LAUTER, J. L. (1982). Dichotic identification of complex sounds: Absolute and relative ear advantages. Journal of the Acoustical Society of America, 7l, 701-707.

LAUTER, J. L. (1983). Stimulus characteristics and relative ear advantages: A new look at old data. Journal of the Acoustical Society of America, 74, 1-17.

LAUTER, J. L. (1984). Contralateral interference and ear advantages for identification of three-element patterns. Brain \& Cognition, 3, 259-280.

Lauter, J. L. (1992). Processing asymmetries for complex sounds: Comparisons between behavioral ear advantages and electrophysiological asymmetries based on quantitative electroencephalography (qEEG). Brain \& Cognition, 19, 1-20.

LAUTER, J. L. (1995). Visions of speech and language: Noninvasive imaging techniques and their applications to the study of human communication. In H. Winitz (Ed.), Human communication and its disorders, a review (Vol. IV, pp. 277-390). Timonium, MD: York Press.

LAUTER, J. L. (1998a). Neuroimaging and the trimodal brain: Implications for developmental communication neuroscience. Folia Phoniatrica et Logopedica, 50, 118-145.

LAUTER, J. L. (1998b). Neurophysiological self-control: Modulation in all things. Journal of Communication Disorders, 31, 543-550.

LAUTER, J. L. (1999a). Fetal nicotine syndrome (FNS): A new explanation for an old problem. Paper presented to Oklahoma Center for Neuroscience Research Symposium on The Neurobiology of Addiction: Neural, Behavioral, and Clinical Features, Oklahoma City, $\mathrm{OK}$.

LAUTER, J. L. (1999b). The handshaking model of brain function: Notes toward a theory. Medical Hypotheses, 52, 435-445.

LAUTER, J. L. (2000). "Central neurolaryngology": Central correlates of within-subject fluctuations in peripheral physiology measured in voice, ear, and heart. Unpublished manuscript.

LAUTER, J. L. (in press). Functional asymmetries and the trimodal brain: Implications for developmental and learning disorders. Journal of Developmental \& Learning Disorders.
Lauter, J. L., Brand, J., Messina, P., \& Perkins, V. (1998). Neural correlates of temporomandibular joint (TMJ) pain. Paper presented to the Oklahoma Center for Neuroscience Symposium, Oklahoma City.

Lauter, J. L., Herscovitch, P., Formby, C., \& Raichle, M. E. (1985). Tonotopic organization in human auditory cortex revealed by positron emission tomography. Hearing Research, 20, 199-205.

Lauter, J. L., Herscovitch, P., \& Raichle, M. E. (1988). Human auditory physiology studied with positron emission tomography. In J. Syka \& R. B. Masterton (Eds.), Auditory pathway (pp. 313-317). New York: Plenum.

LAUTER, J. L., \& KARZON, R. G. (1990). Individual differences in auditory electric responses: Comparisons of between-subject and withinsubject variability: IV. Latency-variability comparisons in early, middle, and late responses. Scandinavian Audiology, 19, 175-182.

LAUTER, J. L., \& LoOMIS, R. L. (1986). Individual differences in auditory electric responses: Comparisons of between-subject and withinsubject variability: I. Absolute latencies of brainstem vertex-positive peaks. Scandinavian Audiology, 15, 167-172.

LAUTER, J. L., LoRD-MAES, J., \& BALDWIN, C. (1999). Repeated evoked potentials (REPs) in multiple sclerosis: Demonstration of a new tool for individual neurological assessment. Tejas, 23, 48-55.

Lauter, J. L., Lynch, O., Wood, S. B., \& Schoeffler, L. (1999). Physiological and behavioral effects of an antivertigo antihistamine in adults. Perceptual \& Motor Skills, 88, 707-732.

LAUTER, J. L., \& OYLER, R. F. (1992). Latency stability of auditory brainstem responses in children aged 10-12 years compared with younger children and adults. British Journal of Audiology, 26, 245-253.

Lauter, J. L., \& Plante, E. (1992). Quantitative electroencephalographic ( $q E E G$ ) correlates of anatomical asymmetries in human auditory cortex studied in the CNS Project. Paper presented to the Society for Neuroscience, Anaheim, CA.

Lauter, J. L., Richey, H., Gilmore, S., \& Lynch, O. (1998). Putting the "central" back in central auditory processing. Journal of Developmental \& Learning Disorders, 2, 51-106.

LAUTER, J. L., Solomon, N., \& LoRD-MAES, J. (1991). MacCAD, a new Macintosh-based HyperCard program for central auditory diagnostics: Description and preliminary findings. Journal of the Acoustical Society of America, 89, 1975.

LiNDAMOOD, C., \& LiNDAMOOD, P. (1979). Lindamood auditory conceptualization ( $L A C$ ) test. Austin, TX: PRO-ED.

Lindamood, P., Bell, N., \& Lindamood, P. (1997). Achieving competence in language and literacy by training in phonemic awareness, concept imagery and comparator function. In C. Hulme \& M. Snowling (Eds.), Dyslexia: Biology, cognition and intervention (pp. 212-234). London: Whurr.

NyBORG, H. (1994a). Hormones, sex and society: The science of physicology. Westport, CT: Praeger.

NyBORG, H. (1994b). The neuropsychology of sex-related differences in brain and specific abilities. In P. A. Vernon (Ed.), The neuropsychology of individual differences (pp. 60-113). San Diego: Academic Press.

Plante, E., Swisher, L., Vance, R., \& Rapcsak, S. (1991). MRI findings in boys with specific language impairment. Brain \& Language, 41, 52-66.

Porges, S. W. (1995). Orienting in a defensive world: Mammalian modifications of our evolutionary heritage. A polyvagal theory. Psychophysiology, 32, 301-318.

Probst, R., Lonsbury-Martin, B. L., \& Martin, G. K. (1991). A review of otoacoustic emissions. Journal of the Acoustical Society of America, 89, 2027-2067.

WitTLING, W. (1995). Brain asymmetry in the control of autonomicphysiologic activity. In R. J. Davidson \& K. Hugdahl (Eds.), Brain asymmetry (pp. 305-357). Cambridge, MA: MIT Press.

ZaPPULLA, R. A. (1991). Fundamentals and applications of quantified electrophysiology. In R. A. Zapulla (Ed.), Windows on the brain: Neuropsychology's technological frontiers (Annals of the New York Academy of Sciences, Vol. 620, pp. 1-21). New York: New York Academy of Sciences.

(Manuscript received December 18, 1998, revision accepted for publication October 3, 1999.) 